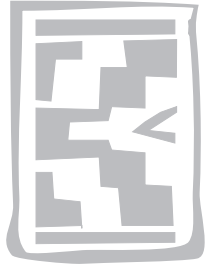

\title{
Prevalence of bovine cysticercosis and hydatidosis in Jimma municipal abattoir, South West Ethiopia
}

\author{
T. TOLOSA ${ }^{1}$, W. TIGRE${ }^{1}$, G. TEKA ${ }^{1}$ and P. DORNY², 3*
}

\begin{abstract}
TOLOSA, T., TIGRE, W., TEKA, G. \& DORNY, P. 2009. Prevalence of bovine cysticercosis and hydatidosis in Jimma municipal abattoir, South West Ethiopia. Onderstepoort Journal of Veterinary Research, 76:323-326

A cross-sectional study to determine the prevalence of bovine cysticercosis and hydatidosis was conducted from October 2007 to March 2008 in cattle slaughtered at the Jimma municipal abattoir. Cyst distribution and viability of bovine cysticercosis and hydatidosis were also determined. A total of 512 carcasses were inspected of which $15(2.93 \%)$ and $161(31.44 \%)$ were infected with Taenia saginata metacestodes and hydatid cysts, respectively. From a total of 109 cysticerci collected from infected carcasses, $47(43.12 \%)$ were viable. The anatomical distribution of the cysticerci was, shoulder muscle $(39.5 \%)$, heart $(33.9 \%)$, neck muscle $(13.8 \%)$, tongue $(10.1 \%)$, masseter muscles $(1.8 \%)$ and diaphragm $(0.9 \%)$. Of the 1171 hydatid cysts collected $223(19.0 \%)$ were fertile, 505 $(43.1 \%)$ sterile, $49(29.8 \%)$ calcified and $94(8.0 \%)$ contained pus. A greater proportion of fertile cysts were found in the lungs than in other organs. It was concluded that these zoonotic cestodes deserve due attention to safeguard public health, and that further studies are needed on genotyping, epidemiology and public health importance of Echinococcus granulosus in the study area.
\end{abstract}

Keywords: Cattle, cysticercosis, Echinococcus granulosus, Ethiopia, hydatidosis, prevalence, Taenia saginata

\section{INTRODUCTION}

Taeniosis and hydatidosis are parasitic zoonoses that present major public health problems in lower income countries (Taylor, Coop \& Wall 2007). Bovine cysticercosis, caused by the metacestodes

* Author to whom correspondence is to be directed. E-mail: pdorny@itg.be

1 Jimma University, College of Agriculture and Veterinary Medicine, School of Veterinary Medicine, P.O. Box, 307, Jimma, Ethiopia

2 Laboratory of Parasitology, Faculty of Veterinary Medicine, Ghent University, Salisburylaan 133, 9820 Merelbeke, Belgium

3 Department of Animal Health, Institute of Tropical Medicine, Nationalestraat 155, 2000 Antwerpen, Belgium

Accepted for publication 16 March 2009-Editor of Taenia saginata is cosmopolitan and occurs in developing as well as in industrialized countries (Dorny, Phiri, Gabriel, Speybroeck \& Vercruysse 2002). The prevalence is high in some African countries (Over, Jansen, Van OIm 1992). In Ethiopia, the prevalence of bovine cysticercosis ranges from 3.1 $\%$ in the central part to $26.25 \%$ in the southern part of the country (Tembo 2001; Dawit 2004; Nigatu 2004; Hailu 2005; Abunna, Tilahun, Megersa, Regassa \& Kumsa 2008). The prevalence of human taeniosis, caused by $T$. saginata, ranges from $10 \%$ to $70 \%$. The tradition of eating raw beef is a major risk factor contributing to this high prevalence (Mamo 1988). Hydatidosis, caused by the metacestodes of Echinococcus granulosus, is endemic in Ethiopia and studies from various parts of the country indicate that the infection ranges from $25.7 \%$ to $63 \%$ and $4.4 \%$ to $18.8 \%$ in cattle and sheep, respec- 
tively (Tamene 1986; Abduljewad 1988; Mohammed 1988; Woubet 1988; Yilkal 1989; Fikre 1994; Mussie 1995). In Jimma town, in the southwestern part of Ethiopia, the hygienic conditions are poor and raw beef consumption is common. Backyard slaughtering of domestic animals, particularly, sheep and goats; and feeding stray dogs with condemned organs are common practices (Abduljewad 1988). These habits promote the transmission of both cestode infections. Hence, this study was conducted with the objective of determining the prevalence of bovine cysticercosis and hydatidosis, slaughtered in the municipal abattoir of Jimma.

\section{MATERIALS AND METHODS}

The study was conducted in the municipal abattoir of Jimma in the southwestern part of Ethiopia, 352 $\mathrm{km}$ southwest (SW) of Addis Ababa. Mixed crop and livestock, in an extensive system, are the main production systems practised in the area. Several species of livestock are reared in the zone, but cattle are the most predominant. They are used as assets, for draft power, meat, milk and hides. Animals are sold only when they become less productive in terms of milk production or draft power. The most common con straints to livestock production are related to communal grazing and watering, poor shelter and underfeeding.

A cross sectional study on bovine cysticercosis and hydatidosis was conducted from October 2007 to
March 2008 on randomly selected animals slaughtered at the Jimma municipal abattoir. The study animals originated from different districts of the Jimma region. Sex, age, breed and origin of each animal were recorded. During meat inspection, carcasses and their respective organs were carefully examined in accordance to the procedures of the Ethiopian Ministry of Agriculture Meat Inspection Regulation (1972) for the detection of $T$. saginata cysticercosis and hydatidosis. Visual inspection and palpation, followed by multiple incisions in the liver, kidney, lung, kidney, heart, diaphragm, shoulder, tongue and masseter muscle, were made to detect the presence of cysticerci and hydatid cysts. Cysts were carefully removed from the carcass and organs and transported to the Parasitology Laboratory of the School of Veterinary Medicine, Jimma University, for further examination.

The viability of $T$. saginata cysticerci was assessed by incubating the cysts in a normal saline solution containing $30 \%$ ox bile at $37^{\circ} \mathrm{C}$ for $2 \mathrm{~h}$. The cysts were regarded as viable if the scolex evaginated during the incubation period. The evaginated cysticerci were examined microscopically for species identification (Gracey, Collins \& Huey 1999).

Hydatid cysts were carefully incised and designated as fertile if protoscolices were found. Fertile cysts were subjected to a viability test (MacPherson, Zeyhle \& Roving 1985) and infertile hydatid cysts were classified as sterile or calcified (Soulsby 1982).

TABLE 1 Taenia saginata cysticercosis in cattle slaughtered at Jimma municipal abattoir $(n=512)$ : Infection levels in different organs

\begin{tabular}{|l|l|l|}
\hline Organs & Range of number of cysticerci recovered & Total/prevalence \\
\hline Shoulder muscle & $1-10$ & $43(39.45)$ \\
Heart & $1-10$ & $37(33.94)$ \\
Tongue & $2-3$ & $11(10.10)$ \\
Masseter muscle & $1-2$ & $2(1.83)$ \\
Neck muscle & $2-5$ & $15(13.76)$ \\
Diaphragm & 1 & $1(0.92)$ \\
\hline Total & $109(100)$ \\
\hline
\end{tabular}

TABLE 2 Viability of hydatid cysts according to the affected organs from cattle slaughtered at the Jimma municipal abattoir $(n=512)$

\begin{tabular}{|c|c|c|c|c|c|}
\hline Organ inspected & Fertile & Sterile & Calcified & Pus formation & Total \\
\hline Lung & $174(21.16)$ & $366(44.52)$ & $219(26.64)$ & $63(7.66)$ & 822 \\
\hline Liver & $38(12.46)$ & 109 (35.74) & $127(41.64)$ & $31(10.16)$ & 305 \\
\hline Spleen & $5(26.32)$ & $14(73.68)$ & $0(0)$ & $0(0)$ & 19 \\
\hline Heart & $4(22.22)$ & $11(61.11)$ & $3(16.67)$ & $0(0)$ & 18 \\
\hline Kidney & $2(28.57)$ & $5(71.43)$ & $0(0)$ & $0(0)$ & 7 \\
\hline Total & 223 (19.04) & 505 (43.13) & $349(29.80)$ & $94(8.03)$ & 1171 \\
\hline
\end{tabular}




\section{RESULTS}

All 512 cattle inspected were adult males and from similar agro-ecological sites and husbandry systems. Of those, 15 (2.93\%) were infected with cysticerci. A total of 109 cysticerci were collected and occurred, in decreasing order, in the shoulder muscle (43), heart (37), neck muscle (15), tongue (11), masseter muscle (2) and diaphragm (1) (Table 1). Forty-seven (43.12\%) were viable and 62 (56.88 $\%)$ were degenerated or calcified. Viable cysticerci were observed in the shoulder (21), cardiac (11), neck (8), tongue (6) and diaphragm (1) muscles, in descending order. The degenerated or calcified cysts were recovered from heart (26), shoulder (22), neck (7), tongue (5), and masseter (2) muscles, in decreasing order.

Out of a total of 512 cattle carcasses, 161 (31.44\%) were infected with hydatid cysts, a total of 1171 hydatid cysts being collected from the infected animals. Of these cysts, $223(19.04 \%)$ were fertile, $505(43.13 \%)$ sterile, 349 (29.80\%) calcified and $94(8.03 \%)$ contained pus (Table 2). Cysts were found in the lungs (57\%), liver (34\%), spleen (5\%), heart (3\%) and kidneys (1\%).

\section{DISCUSSION}

In this study the prevalence of cysticercosis was $2.93 \%$, which is comparable to reports on the subject emanating from central Ethiopia (3.11\%) (Tembo 2001), but lower than that reported in other parts of the country (Dawit 2004; Nigatu 2004; Hailu 2005; Abunna et al. 2008). The different prevalences reported in these studies might be due to several factors of which husbandry systems, hygiene differences and eating habits are among the most important. In addition, diagnosis of bovine cysticercosis by meat inspection underestimates the true prevalence, especially when infection is light (Dorny \& Praet 2007). The higher prevalence of cysticercosis in developing countries is associated with poor sanitary infrastructure, low awareness and improper disposal of sewage, which also pertains to Ethiopia, where the widespread habit of eating raw meat is an additional important risk factor.

The high prevalence of hydatidosis of $31.45 \%$ is in accord with findings in other parts of the country. Most of the slaughtered animals were adults, which were most likely culled due to inefficiency for draught purpose. Old animals are likely to have a higher possibility of acquiring infection due to their longer exposure to infection and to lower immunity to com- bat infection. In this study, the lungs were the most frequently affected organ (57\%), followed by the liver (34\%), which is in agreement with other studies in cattle in Ethiopia (Mohammed 1988; Abduljuwad 1988). Also, a much higher proportion of fertile cysts was observed in the lungs than in any other organ. The fertility rate of hydatid cysts may increase with advancing age of the hosts due to reduced immunological compatibility of animals (Himonas 1987). The prevalence of hydatidosis is likely to be high in Ethiopia owing to several factors favouring transmission, among which are, keeping of dogs in close association with ruminants, low public awareness on the role of offal in transmitting hydatid cysts, feeding of dogs with hydatid infected organs and backyard slaughtering practices. In addition, Jimma town has a large population of stray dogs that visit the abattoir grounds to be fed with condemned organs (personal observations).

To reduce infections of these cestodes to cattle and prevent transmission to humans attention should be given to such considerations aspersonal hygiene, meat hygiene, control of stray dog populations and cestodicidal treatment of dogs in the study area. While taeniosis in humans has generally little clinical significance, hydatid disease is a serious condition that warrants control measures to be taken. In order to assess the zoonotic potential of $E$. granulosus, genotyping of parasites isolated from cattle, sheep and goats should be undertaken.

\section{ACKNOWLEDGEMENTS}

This work was supported by the Flemish Inter University Council (VLIR-Jimma University IUC Programme Ethiopia-Belgium). The cooperation of Jimma municipal abattoir is appreciated.

\section{REFERENCES}

ABDULJEWAD, A. 1988. Hydatidosis prevalence at Jimma Abattoir. D.VM. thesis, Faculty of Veterinary Medicine, Addis Ababa University.

ABUNNA, F., TILAHUN, G., MEGERSA, B.A., REGASSA, A. \& KUMSA, B. 2008. Bovine cysticercosis in cattle slaughtered at Awassa municipal abattoir, Ethiopia: Prevalence, cyst viability, distribution and its public health implication. Zoonoses and Public Health, 55:82-88.

DAWIT, S. 2004. Epidemiology of T. saginata taeniasis and cysticercosis in North Gondor Zone. DVM thesis, Faculty of Veterinary Medicine, Addis Ababa University.

DORNY, P., PHIRI, I., GABRIEL, S., SPEYBROECK, N. \& VERCRUYSSE, J. 2002. A sero-epidemiological study of bovine cysticercosis in Zambia. Veterinary Parasitology, 104: 211215. 
DORNY, P. \& PRAET, N. 2007. Taenia saginata in Europe. Veterinary Parasitology, 149:22-24.

FIKRE, L. 1994. Echinococcosis/Hydatidosis in Konso (Southern Ethiopia): An assessment trial of its prevalence, economic and public health importance. D.VM. thesis, Faculty of Veterinary Medicine, Addis Ababa University.

GRACEY, J.F., COLLINS, D.S. \& HUEY, R.J. 1999. Meat hygiene, $10^{\text {th }}$ ed. London: Harcourt Brace \& Company.

HAILU, D. 2005. Prevalence and risk factors for Taenia saginata cysticercosis in three selected areas of Eastern Shoa. M.Sc. thesis, Faculty of Veterinary Medicine, Addis Ababa University.

HIMONAS, C. 1987. The fertility of hydatid cysts in food animals in Greece. Helminth Zoonoses. Amsterdam, The Netherlands: Martinus Nijhoff Publishers.

MACPHERSON, N.L., ZEYHLE, E. \& ROVING, T. 1985. An echinococcosis pilot control programme for north west Turkana, Kenya. Annals of Tropical Medicine and Parasitology, 78: 188-192.

MAMO, E. 1988. Some common zoonotic helminths, in The ecology of health, edited by A.Z. Zein \& H. Kool, Ministry of Health, Addis Ababa.

MINISTRY OF AGRICULTURE 1972. Meat inspection regulations. Legal Notice No. 428 Negarite Gazexa, Addis Ababa.

MOHAMMED, A. 1988. Study on prevalence and economic significance of bovine hydatidosis in Gamo-Goffa region. D.VM. thesis, Faculty of Veterinary Medicine, Addis Ababa University.

MUSSIE, H. 1995. Bovine hydatid disease: an assessment trial of its prevalence and economic importance at Bahir Dar slaughterhouse. D.VM. thesis, Faculty of Veterinary Medicine, Addis Ababa University.

NIGATU, K. 2004. Cysticercus bovis: development and evaluation of serological tests and prevalence at Addis Ababa abattoir. M.Sc. thesis, Faculty of Veterinary Medicine, Addis Ababa University.

OVER, H.J., JANSEN, J. \& VAN OLM, P.W. 1992. Distribution and impact of helminth diseases of livestock in developing countries. Rome, Italy: FAO (Animal Production and Health Paper, 96).

SOULSBY, E.J.L. 1982. Helminths, arthropods and protozoa of domesticated animals, $7^{\text {th }}$ ed. London: Bailliere Tindall.

TAMENE, M. 1986. A preliminary study on echinococosis/hydatidosis of livestock (cattle, sheep and goats) in Gondar administrative region. D.VM. thesis, Faculty of Veterinary Medicine, Addis Ababa University.

TAYLOR, M.A., COOP, R.L. \& WALL, R.L. 2007. Veterinary parasitology, $3^{\text {rd }}$ ed. Oxford: Blackwell Publishing.

TEMBO, A. 2001. Epidemiology of Taenia saginata taeniasis and cysticercosis in three selected agro- climatic zones in central Ethiopia. S.Sc. thesis, Faculty of Veterinary Medicine, Addis Ababa University \& Free University of Berlin.

WOUBET, S. 1988. Prevalence of cattle hydatidosis and its economic significance in Robe Municipality abattoir. D.VM. thesis, Faculty of Veterinary Medicine, Addis Ababa University.

YILKAL, A. 1989. Hydatidosis in cattle, sheep, pigs; Cysticercus tenuicollis in sheep around Dessie and the efficacy of $\mathrm{Ha}$ genia abyssinica (Kosso) on Taenia hydatigena. D.VM. thesis, Faculty of Veterinary Medicine, Addis Ababa University. 